\title{
A Note on the Application of Pulse Compression Tech- niques to Ionospheric Sounding
}

\author{
D. C. Coll and J. R. Storey
}

\author{
Department of National Defence, Defence Research Board, Defence Research Telecommunications \\ Establishment, Ottawa 4, Ontario, Can.
}

(Received October 5, 1964)

\begin{abstract}
Ionograms are presented which demonstrate that significant improvements in signal-to-noise can be obtained by replacing the single pulses of a conventional sounder by a coded-pulse signal.
\end{abstract}

In an earlier paper [Coll and Storey, 1964] ${ }^{1}$ techniques for the application of pulse compression to ionospheric sounding were discussed. In that paper, the generation and reception of coded-pulse signals were described and some preliminary results were presented. Since that time, the experimental equipment has been extended so that swept-frequency ionospheric sounding could be carried out over oblique paths. It is the purpose of this note to present ionograms that demonstrate that significant improvements in signal-to-noise ratio can be obtained, without degradation of frequency information, by replacing the single pulses of a conventional sounder by coded-pulse signals.

The use of coded-pulse, or pulse compression, signals is one way of increasing the energy transmitted in sounding signals which is particularly suited for use with transmitters that are peak-power limited. Essentially, the use of coded-pulse transmissions provides the energy increase associated with lengthened pulses while retaining the range resolution of a narrow pulse.

The signals used in these experiments consisted of binary, and impulse-equivalent, pulse trains, and single pulses with the same peak power. The generation and detection of these pulse sequences was described in the previous paper.

The sounding system is shown in figure 1. The pertinent parameters used were as follows:

(i) frequency range: 3 to $16 \mathrm{MHz}$.

(ii) frequency increment: $10 \mathrm{kHz}$.

(iii) frequency increments per second: 10 .

(iv) transmissions per second: 10 coded pulsesingle pulse pairs.

(v) peak power: $1 \mathrm{~kW}$.

(vi) single pulse length: $25 \mu$ sec.

(vii) length of coded-pulses: $425 \mu$ sec. (17 component pulses).

${ }^{1}$ Coll, D. C., and J. R. Storey (Oct. 1964), Ionospheric sounding using coded-pulse signals, Radio Sci. J. Res. NBS 68D, No. 10, 1155-1159.

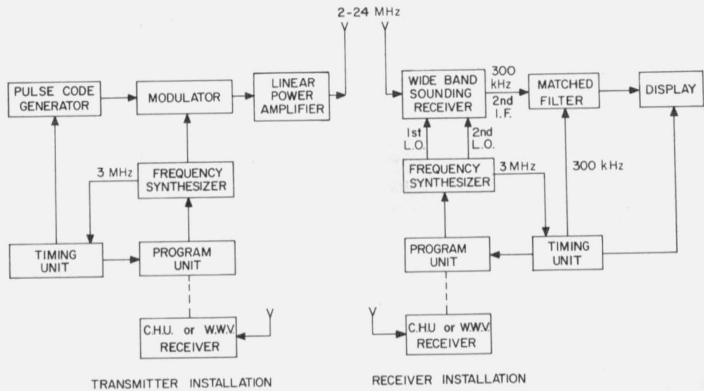

FigurE 1. Oblique ionospheric sounding system.

For contrast, and demonstration purposes, each coded-pulse transmission was followed by a single pulse transmission and separate ionograms were produced from each. The displays were qualitatively adjusted by personnel experienced in the production of ionograms, to achieve a balance between signals and noise. The sounding trials were carried out over a $1000 \mathrm{~km}$ path between Ottawa, Ont., and Halifax, N.S.

The advantage of a binary pulse train over a single pulse is demonstrated in figure 2. Two features are of interest: the clarity with which the $F_{2}-$ MUF may be seen, and the 2-hop $F_{2}$ propagated signal apparent between 3 and $5 \mathrm{MHz}$.

The advantage of an impulse-equivalent, or Huffman, pulse train over a single pulse is demonstrated in figure 3 . The improvement in signal-to-noise ratio is again significant. In this case there are two 2-hop traces.

As an added experiment [Coll, Storey, and Pearce, $1965]^{2}$ the normal matched filter was replaced by the digital matched filter shown in figure 4. A comparison between the ionogram produced by the analog matched filter and that produced by the digital matched filter

${ }^{2}$ Coll, D. C., J. R. Storey, and H. M. Pearce (Feb. 1965), Digital detection of coded-pulse ionosonde signals, Proc. IEEE 53, No. 2, 188-189. 


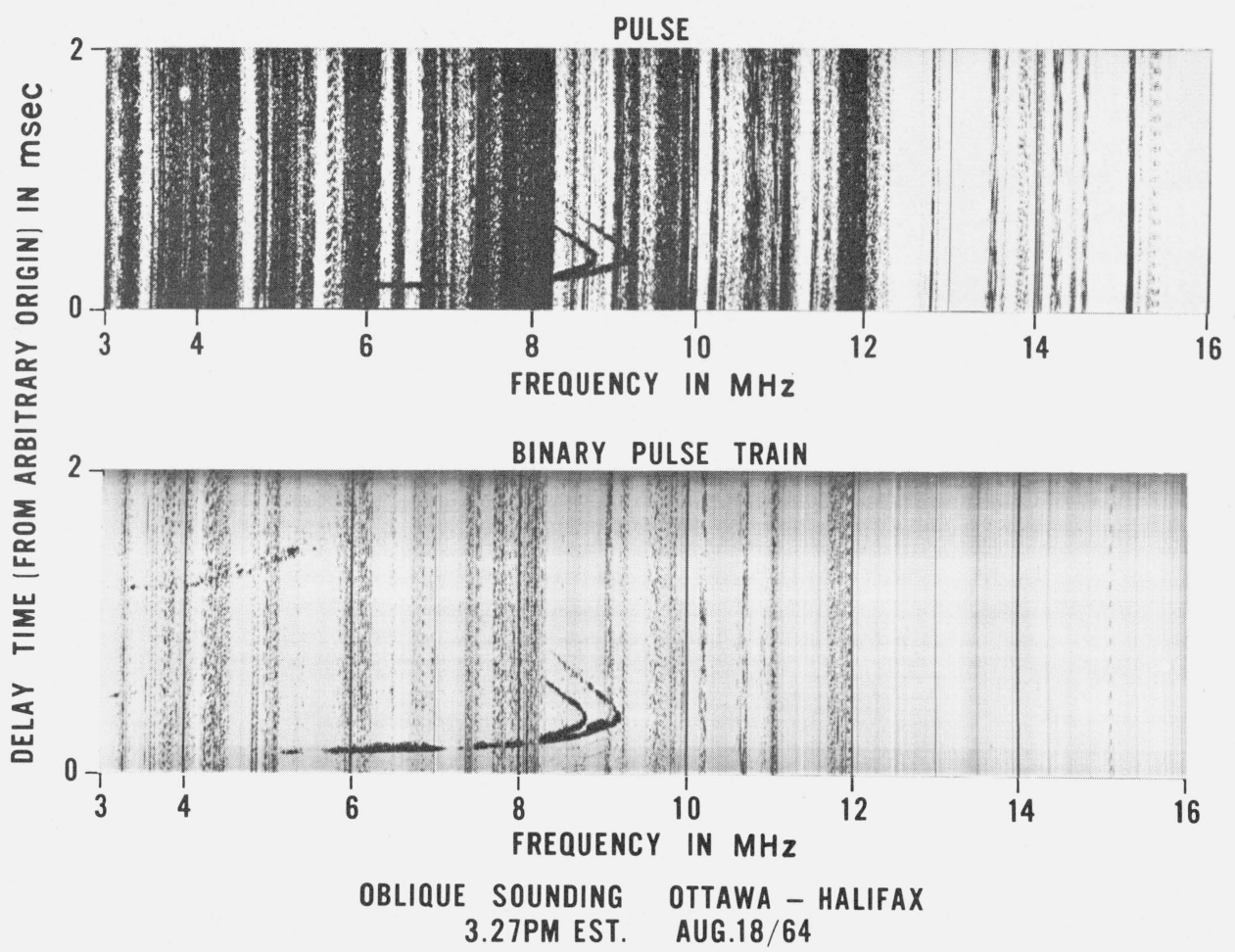

FIGURE 2. Binary pulse train versus single pulse with the same peak power.

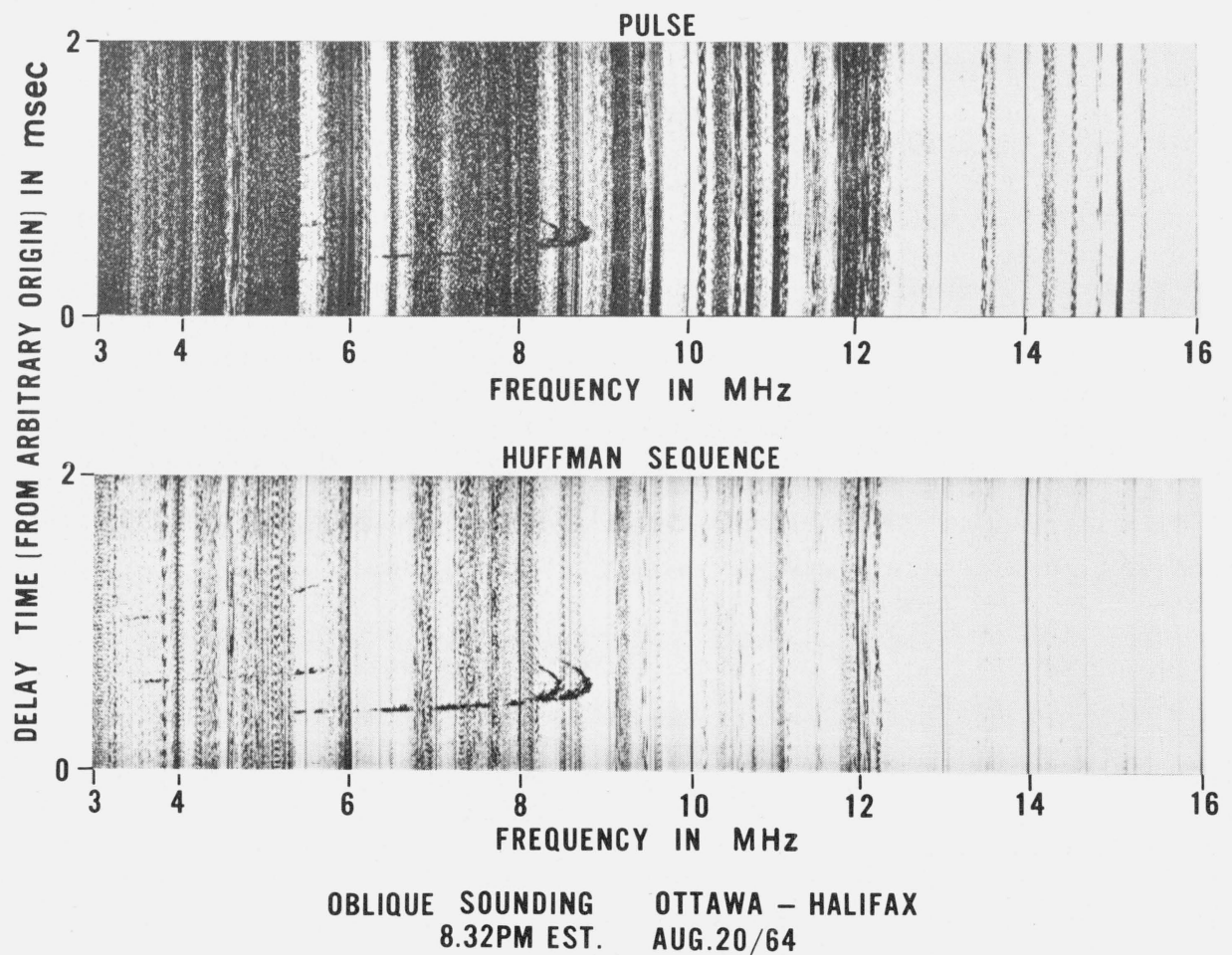

FIGURE 3. Impulse equivalent pulse train (Huffman signal) versus single pulse. 


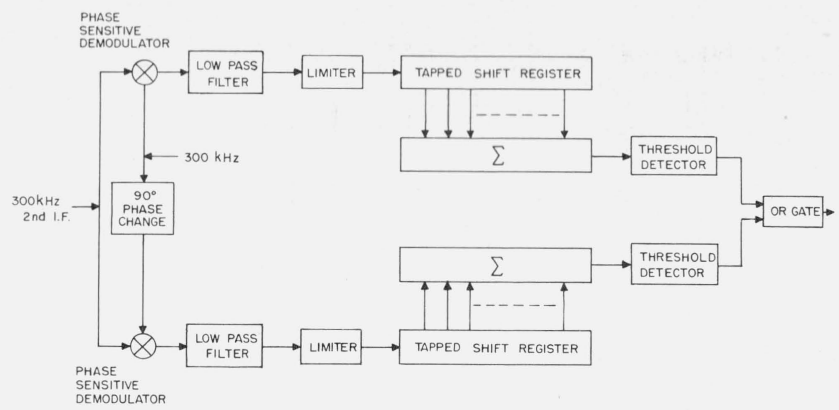

FIGURE 4. Digital quadrature matched filter detector.

is presented in figure 5. There are several points about the digital reception:

(i) There is a constant false alarm rate, i.e., the noise produces a speckled background. The eye is able to ignore this noise in favor of signals, and simple digital processing could remove most of it without disturbing the signal.

(ii) There is an increased probability of detection, as shown by the signals apparent between 4.5 and 6.5 $\mathrm{MHz}$.

(iii) The characteristic bands of noise, representing powerful signals, do not appear on the digital ionogram. The result is pleasing to the eye, however the high noise on these frequencies tends to destroy the signals, and the effect is equivalent to a fast-acting AGC or squelch.

(iv) The ionogram is produced by digital (two-level) signals. There are no problems with display saturation, and the digital signals may be fed directly to a digital computer for further processing and analysis.

(v) The digital reception may be used for the display of frequency information on ionograms. If amplitude information is desired, it may be obtained from the output of an analog matched filter.

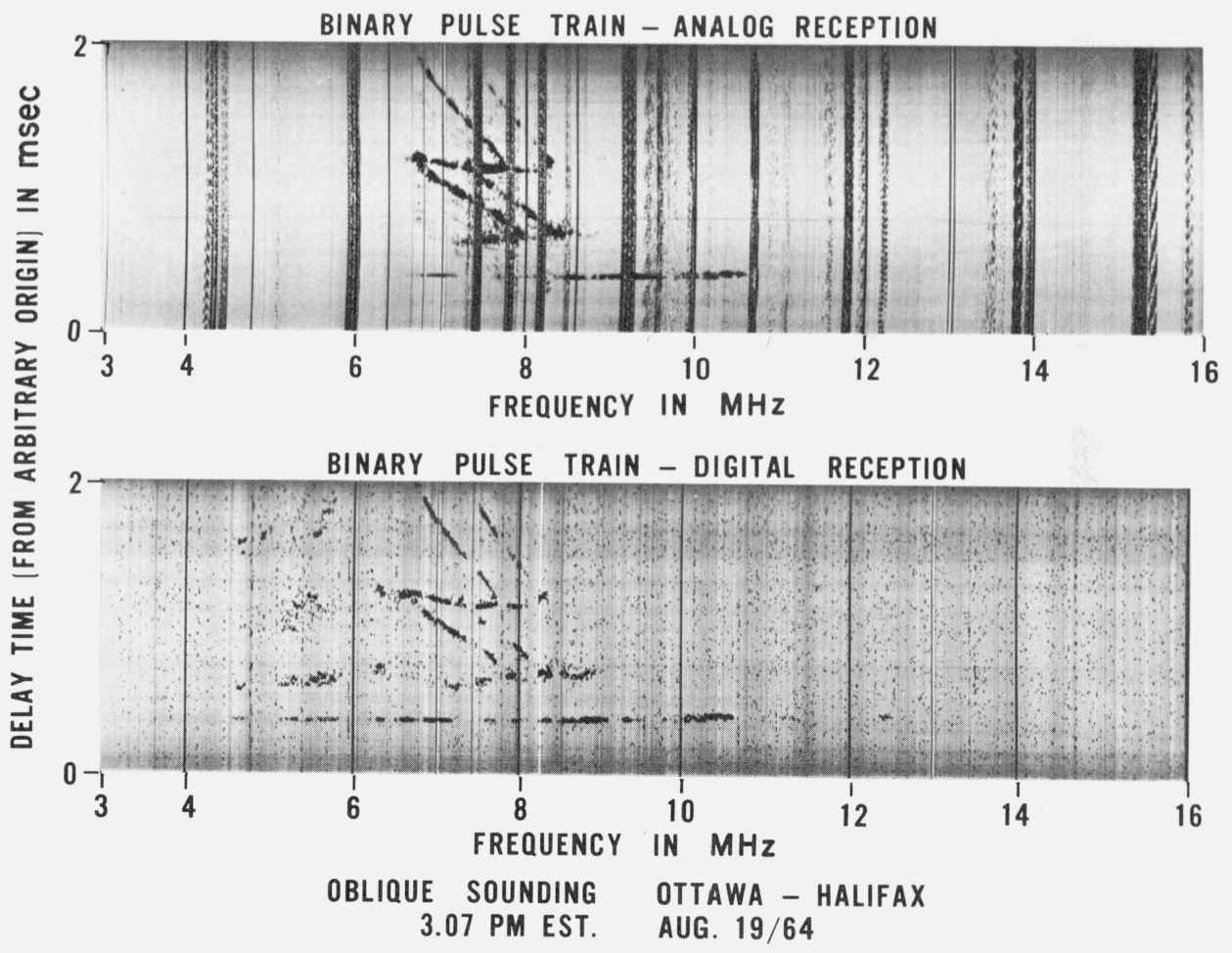

FigURE 5. Analog versus digital reception of binary pulse train. 